\title{
A case-control study of the periodontal condition in gastric cancer patients
}

\author{
Nikolaos Andreas Chrysanthakopoulos ${ }^{1,2}$, Aggelos Antonios Oikonomou ${ }^{3}$ \\ ${ }^{1} 401$ General Military Hospital of Athens, 11525 Athens, Greece. \\ ${ }^{2}$ Department of Pathological Anatomy, Medical School, University of Athens, 11527 Athens, Greece. \\ ${ }^{3}$ Internal Medicine, Private Practice, 26221 Patra, Greece.
}

Correspondence to: Dr. Nikolaos Andreas Chrysanthakopoulos, Department of Pathological Anatomy, Medical School, University of Athens, 11527 Athens, Greece. E-mail: nikolaos_c@hotmail.com; nchrysant@med.uoa.gr

How to cite this article: Chrysanthakopoulos NA, Oikonomou AA. A case-control study of the periodontal condition in gastric cancer patients. Stomatological Dis Sci 2017;1:55-61.

Prof. Nikolaos Andreas Chrysanthakopoulos was graduated from Dental School, University of Athens in 1998. He
has also received another Degree from Faculty of Nursing from University of Athens in 1993. He was attended a
program in Maxillofacial and Oral Surgery in 401 General Military Hospital of Athens during 2003-2004. He got
specialist in Clinical Oncology, Cytology and Histopathology during 2013-2015. His MSc Thesis titled "Correlation
Between Periodontal Disease Indices and Lung Cancer in Greek Adults: a Case-Control study" consists the first $\$$
in the International literature that was examined the possible association between periodontal diseases and lung
cancer. Prof. Chrysanthakopoulos is a 6th year Pre-Grdt Student in Medical School, University of Athens. He is also
a Professor of Human Anatomy, Human Physiology and Sports Medicine in Public Institute of Vocational Training $\$$
of Patra. He has published over 45 manuscripts in International Medical and Dental Journals as unique or the 1st author. He is Editorial
Board Member in 9 International Medical and Dental Journals, Associate Editor in 1 International Medical Journal and Reviewer in
34 International Medical and Dental Journals. He has received invitations for writing Chapters in several e-Books regarding Dental
Anatomy, Oral Health and Periodontics and to participate in International Medical and Dental Congresses as a Speaker.

\section{Article history:}

Received: 06-02-2017

Accepted: 12-04-2017

Published: 29-06-2017

Key words:

Gastric cancer,

periodontal disease,

adults,

risk factors

\section{ABSTRAC T}

Aim: The present study was carried out to examine the possible differences regarding the periodontal condition between individuals with a gastric cancer history diagnosed by histological examination and healthy individuals. Methods: Sixty-five patients suffered from non-cardia adenocarcinoma diagnosed by histological examination and 60 matched healthy controls were interviewed and clinically examined. Data analysis was performed by $\chi^{2}$ test and logistic regression model to estimate possible correlations regarding the periodontal condition of the mentioned individuals after assessment. The assessment included the following clinical parameters: probing pocket depth, clinical attachment loss, gingival index, and bleeding on probing. Results: Smoking [odds ratio $(\mathrm{OR})=4.448,95 \%$ confidence interval $(\mathrm{CI})=1.818$ 10.880], gingival inflammation $(\mathrm{OR}=2.019,95 \% \mathrm{CI}=0.933-11.579)$ and clinical attachment loss $(\mathrm{OR}=5.167,95 \% \mathrm{CI}=2.122-12.584)$, were statistically significant differences between the cases and the controls, after adjustment for smoking and socioeconomic status. Conclusion: Smoking, gingival inflammation, and clinical attachment loss were statistically significantly different between patients who had gastric cancer and healthy individuals.

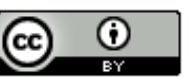

This is an open access article licensed under the terms of Creative Commons Attribution 4.0 International License (https://creativecommons.org/licenses/by/4.0/), which permits unrestricted use, distribution, and reproduction in any medium, as long as the original author is credited and the new creations are licensed under the identical terms.

For reprints contact: service@oaepublish.com

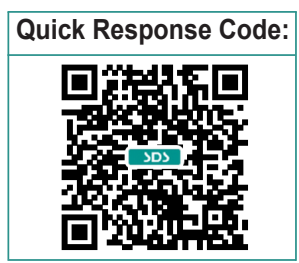




\section{INTRODUCTION}

Gastric cancer (GC) constitutes a fatal disease, which is characterized by severe symptoms and clinical signs, and significantly affects the patients' quality of life. ${ }^{[1]}$ Genetic influences, in combination with environmental and behavioral risk factors, have been suggested as pathogenic features of GC. The genetic component includes advanced age, male gender, genetic predisposition, family history of cancer, and other factors that remain unknown. The environmental and behavioral risk factors include heavy smoking, alcohol consumption, dietary habits, and previous $\mathrm{H}$. pylori infection. ${ }^{[2,3]}$ However, the mentioned factors, can only explain a part of the GC incidence. ${ }^{[4]}$

Periodontal disease (PD) especially periodontitis, is a chronic destructive and progressive disease that can affect one or more of the periodontal tissue structures. These structures are the alveolar bone, cementum, periodontal ligament, etc. ${ }^{[5]}$ Periodontal infection contributes to periodontitis and leads to systemic effects. Significant associations have been recorded between PD and systemic disorders; such as cardiovascular and atherosclerotic diseases, respiratory diseases, allergies, diabetes mellitus, etc. ${ }^{[6]}$ In addition, several authors have investigated the possible causative role of periodontitis in cancer development. This cancer development may include in the oral cavity, the esophagus and stomach, and the lungs and pancreas. ${ }^{[7-11]}$ The results are conflicting even after controlling for potential confounders.

On the contrary, a few studies have been completed regarding the oral conditions in GC patients and other types of cancer. However, the possible oral and periodontal tissue conditions may be visible during the progression of the disease and in different appearance in the same patient.

The higher risk of periodontal tissue lesions in patients with cancer has been suggested to be a result of psychological burden rather than disturbances in the patients' nutrition or alterations in the oral cavity regarding the quantity/quality of saliva. Additionally, disturbances in the balance of microbiological and immunological parameters in the oral cavity that could be affected because of the chemotherapy or radiotherapy. ${ }^{[12,13]}$ It is also possible that GC patients are more susceptible to the progression and destruction of periodontal tissue than the healthy population. This observation could be attributed to the extremely poor prognosis of GC.
The present research was carried out to estimate the possible differences regarding the PD condition between individuals with a GC history, diagnosed by histological examinations, and healthy patient.

\section{METHODS}

\section{Study sample}

The sample of the study included 125 individuals, 61 males and 64 females, aged 48-78 years, which were selected from two private practices (a medical practice and a dental practice). The participants completed a medical and dental health questionnaire, and were examined clinically regarding their oral condition. The current study was performed between December 2015 and September 2016.

\section{Patients' selection criteria}

Sixty-five GC patients, who were referred by a private medical and a dental practice, accepted the invitation to take part in the study. The diagnosis of GC was determined by clinical criteria such as symptoms and signs; however, the histopathological procedure was the main diagnostic tool for the definitive diagnosis of GC. Sixty healthy individuals who were referred by a dental practice comprised the control group. Both groups, the cases and the controls, were selected from the same city population in order to avoid possible selection biases. A statistical approach for confounding control was that the selection of the control group was based on the GC patients' environment, such as friends, colleges, etc. To be more specific, the control group and the GC patients were matched regarding their age, gender, and smoking status (current/previous smokers and never smokers). According to epidemiological studies age, ${ }^{[14]}$ smoking history, ${ }^{[15]}$ and gender ${ }^{[16]}$ have been found to be the principle risk factors for periodontitis development as covariates; ${ }^{[17]}$ thus, both groups were matched for the these parameters. For each GC patient, a healthy individual of the same gender and the same age ( \pm 3 years) was selected. For 5 of the 65 cases, no controls were found to match the criteria. The cases and the controls had at least a mean of 20 natural teeth, since less than 20 natural teeth could affect the estimation of the clinical indices examined.

In addition, all of the patients meet the clinical criteria of established periodontitis. ${ }^{[18]}$ All of the participants had not received any periodontal treatment, conservative or surgical, during the previous 6 months. The patients had not been prescribed antibiotics, used anti-inflammatory, or other systemic drugs during the previous 6 weeks. ${ }^{[19]}$ 
To avoid potential confounding influences as much as possible, the following indices were not included: diabetes mellitus, cardiovascular diseases, rheumatoid arthritis, liver cirrhosis, immunosuppressed patients or glucocorticoid therapy. Also excluded were individuals with recurrent or advanced GC, active gastrointestinal bleeding, a history of esophageal varices, prior gastric surgery for cancer, current use of anticoagulants, and gastric metastases due to a different primary location. Patients diagnosed with cardia-adenocarcinoma or other types of cancer which are located in the region of the head-neck-thorax-carcinogenesis field ${ }^{[20]}$ were excluded from the study. The conditions/disorders could have potential effects on the oral tissues.

Similarly, hospital patients or patients with several types of cancer in which smoking is considered as a causal factor or as a risk factor such as larynx cancer, nasopharyngeal cancer, etc. were not included in the study. No oral hygiene instruction was given to the patients' group after diagnosis of GC and before the application of any medical treatment, i.e. surgery, radiotherapy or chemotherapy, as a major problem would have occurred regarding the intra-examiner variance.

\section{Oral clinical examination}

From the medical/dental health questionnaire and the participant's dental records, the following variables were recorded: gingival index $(\mathrm{GI})$, probing pocket depth (PPD), clinical attachment loss (CAL) and bleeding on probing (BOP). All of the permanent teeth were clinically examined (except 3rd molars and any remaining root-tips) using a William's 12 PCP probe (PCP 10-SE, Hu-Friedy Mfg. Co. Inc., Chicago, IL, USA) at six sites per tooth (mesiofacial, distofacial, facial, distolingual, lingual, and mesiolingual) by the assessing dentist.

\section{Gingival index}

Six sites per tooth were examined in order to estimate the presence or absence of gingival inflammation. Using the Löe and Silness classification ${ }^{[21]}$ gingival index was coded as follows: score 0 , no inflammation; score 1, mild inflammation; score 2, moderate inflammation; and score 3, severe inflammation.

\section{Periodontal examination}

Six sites per tooth were also examined in order to estimate the mentioned indices (PPD, BOP, and CAL). In the cases where the Cement-Enamel Junction (CEJ) was obscured by a restoration or the tooth cervix was destructed by abrasion, decay or another lesion, the CEJ location was recorded by extrapolating the CEJ location from the adjacent teeth. In case the CEJ location was not visible, no data was recorded. PPD was coded as follows: ${ }^{[2]}$ score 0 : moderate periodontal pockets, 4-6 $\mathrm{mm}$; and score 1: advanced periodontal pockets, $>6 \mathrm{~mm}$. CAL severity was coded as follows: ${ }^{[23]}$ score 0 : mild, 1-2 mm of attachment loss; and score 1: moderate/severe, $\geq 3 \mathrm{~mm}$ of attachment loss. The PPD and CAL recorded to the immediate full millimeter. BOP absent/present was coded as follows: score 0: BOP absent and score 1: BOP present, which is considered as positive if it occurred within $15 \mathrm{~s}$ of probing.

\section{Questionnaire}

A self-administered questionnaire was completed by the cases and the controls. That questionnaire included the following parameters: age, gender, smoking status, socioeconomic and educational level and information regarding the participants' medical history concerning the mentioned systemic conditions/ disorders, medications and the frequency of their dental follow-up examinations. A random sample was chosen and consisted of $35(20 \%)$ individuals was reexamined clinically by the same dentist after three weeks in order to record the intra-examiner variance. After consideration of the code numbers of the double-examined individuals, no differences were found between the clinical examinations (Cohen's Kappa $=0.98)$.

The current study is a case-control study and could not be reviewed and approved by authorized committees (Greek Dental Associations, Ministry of Health, etc.). However, it was performed in full accordance with the World Medical Association Declaration of Helsinki. Individuals who agreed to be in the study signed an informed consent form.

\section{Statistical analysis}

After dental clinical examinations of the cases and the controls, the worst values of the parameters assessed were recorded and coded as dichotomous variables. Males were coded as 1 , current and former smokers were coded as 1 , subjects with a high socioeconomic (income/monthly equivalent to or above $1,000 €$ ) and educational (graduated from University/College) level were coded as 1 , individuals that had a regular dental follow-up were coded as 1 . Initially $\chi^{2}$ test was performed to estimate the relationship between the independent parameters examined and the GC risk, separately. Secondly, a multivariate regression analysis was performed to examine the associations between GC as a dependent variable and the independent variables were determined by the enter method. Adjusted odds 
ratios (ORs) and 95\% confidence intervals (Cls) were assessed as well. Finally, the independent variables were included in stepwise method to estimate the variables this showed statistically significant associations with the dependent variable. To avoid biased secondary associations, the statistical methods Cochran's and Mantel-Haenszel's test were calculated to control the possible confounders. Statistical analysis was carried out using the statistical package SPSS ver.17.0. A $P$ value less than $5 \%(P<0.05)$ was considered to be statistically significant.

\section{RESULTS}

The participants showed a mean age of $64.3( \pm 3.8)$ years. The univariate analysis is presented in Table 1 regarding the examined variables. Educational level was found to be statistically significantly between the cases and the controls. Table 1 presents the unadjusted OR's and 95\% Cls. According to the step $1 \mathrm{a}$ of the model, the main finding was that smoking and CAL were statistically significant differences between the cases and the controls [Table 2]. Table 2 also presents adjusted ORs with $95 \% \mathrm{Cls}$ and the final step $9 a$ in which smoking, gingival inflammation (GI), and
CAL were significantly differences between the cases and the controls. The findings existed after adjusting for known confounders, smoking and socioeconomic status [Table 3].

\section{DISCUSSION}

This study has limitations that should be considered when interpreting the results. A case-control study does not have the reliability of a prospective study. Additionally, random bias, recall biases and the effect of known and unknown confounders are likely to be higher. Another limitation was that the study information based on the individuals' responses to the questionnaire. Therefore, the participants could not respond to some of the questions or could overestimate or underestimate their potential medical problems. The issues could lead to restrictions on the study's validity during the interpretation of the results. The aim of the present study was to perform a comparison between GC patients and a control group regarding several PD indicators. The aim was not to investigate a possible association between PD indicators, such as causal/risk factors, and GC development. The results showed no statistically significant difference between the groups

Table 1: Univariate analysis of the cases and the controls regarding each independent variable examined, $n$ (\%)

\begin{tabular}{|c|c|c|c|c|c|}
\hline Variables & Cases & Controls & $P$ value & OR & $95 \% \mathrm{Cl}$ \\
\hline \multicolumn{6}{|l|}{ Gender } \\
\hline Males & $36(55.4)$ & $25(41.7)$ & & & \\
\hline Females & $29(44.6)$ & $35(58.3)$ & 0.125 & 1.74 & $0.86-3.53$ \\
\hline \multicolumn{6}{|l|}{ Age (years) } \\
\hline $48-51$ & $7(11.2)$ & $8(15.5)$ & & & \\
\hline $52-61$ & $28(42.5)$ & $23(32.5)$ & & & \\
\hline $62-71$ & $18(31.3)$ & $16(37.7)$ & 0.555 & - & - \\
\hline $72+$ & $12(15.0)$ & $13(14.3)$ & & & \\
\hline \multicolumn{6}{|l|}{ Socio-economic level } \\
\hline Low & 27 (41.5) & 32 (53.3) & & & \\
\hline High & $38(58.5)$ & $28(46.7)$ & 0.187 & 0.62 & $0.31-1.26$ \\
\hline \multicolumn{6}{|l|}{ Educational level } \\
\hline Low & $21(32.3)$ & $34(44.0)$ & & & \\
\hline High & $44(67.7)$ & $26(56.0)$ & $0.006^{*}$ & 0.37 & $0.18-0.76$ \\
\hline \multicolumn{6}{|l|}{ Smoking } \\
\hline No & $28(43.1)$ & $34(56.7)$ & & & \\
\hline Yes & 37 (56.9) & $26(43.3)$ & 0.129 & 0.58 & $0.29-1.18$ \\
\hline \multicolumn{6}{|l|}{ Annual dental follow-up } \\
\hline$<2$ times or no/year & $32(49.2)$ & $28(46.7)$ & & & \\
\hline 2 times/year & $33(50.8)$ & $32(53.3)$ & 0.774 & 1.11 & $0.55-2.24$ \\
\hline \multicolumn{6}{|l|}{ Gingival index } \\
\hline normal/mild inflammation & $27(41.5)$ & $33(55.0)$ & & & \\
\hline medium/severe inflammation & 38 (58.5) & $27(45.0)$ & 0.132 & 0.58 & $0.29-1.18$ \\
\hline \multicolumn{6}{|l|}{ Periodontal pockets } \\
\hline Depth $0-4.0 \mathrm{~mm}$ & $28(43.1)$ & $22(36.7)$ & & & \\
\hline Depth $\geq 5.0 \mathrm{~mm}$ & $37(56.9)$ & $38(63.3)$ & 0.465 & 1.31 & $0.64-2.68$ \\
\hline \multicolumn{6}{|l|}{ CAL } \\
\hline Mild/moderate $0-5.0 \mathrm{~mm}$ & $25(38.5)$ & $24(40.0)$ & & & \\
\hline Severe $\geq 6.0 \mathrm{~mm}$ & $40(61.5)$ & $36(60.0)$ & 0.860 & 0.94 & $0.46-1.92$ \\
\hline \multicolumn{6}{|l|}{$\mathrm{BOP}$} \\
\hline No & $23(35.4)$ & $26(43.3)$ & & & \\
\hline Yes & $42(64.6)$ & $34(56.7)$ & 0.363 & 0.72 & $0.35-1.47$ \\
\hline
\end{tabular}

OR: odds ratio; Cl: confidence interval; PPD: periodontal pockets depth; CAL: clinical attachment loss; Cl: confidence interval; BOP: bleeding on probing; ${ }^{*} P$ : statistically significant (according to chi-square test) 
Table 2: Presentation of correlation between independent variables and gastric cancer according to Enter (first step) and Wald (final step) method of multiple logistic regression analysis model

\begin{tabular}{|c|c|c|c|c|c|c|c|c|}
\hline & & B & Standard error & Wald & Degree of freedom & $P$ value & Expected values (B) & $95 \% \mathrm{Cl}$ for $\operatorname{Exp}(\mathrm{B})$ \\
\hline \multirow[t]{11}{*}{ Step 1a } & gender & 0.157 & 0.421 & 0.140 & 1 & 0.709 & 0.855 & $0.375-1.948$ \\
\hline & age & 0.091 & 0.220 & 0.172 & 1 & 0.678 & 1.095 & $0.712-1.684$ \\
\hline & socioecon_st & 0.391 & 0.498 & 0.618 & 1 & 0.432 & 1.479 & $0.558-3.923$ \\
\hline & educ_lev & 0.420 & 0.453 & 0.859 & 1 & 0.354 & 1.521 & $0.626-3.694$ \\
\hline & smok_stat & 1.504 & 0.539 & 7.795 & 1 & $0.005^{\star}$ & 4.498 & $1.565-12.927$ \\
\hline & dent_foll_up & 0.126 & 0.440 & 0.082 & 1 & 0.774 & 1.135 & $0.479-2.689$ \\
\hline & GI & 0.341 & 0.424 & 0.645 & 1 & 0.422 & 1.406 & $0.612-3.230$ \\
\hline & PPD & 0.858 & 0.460 & 3.476 & 1 & 0.062 & 2.358 & $0.957-5.812$ \\
\hline & CAL & 1.447 & 0.494 & 8.582 & 1 & $0.003^{*}$ & 4.252 & $1.615-11.199$ \\
\hline & $\mathrm{BOP}$ & 0.039 & 0.433 & 0.008 & 1 & 0.929 & 0.962 & $0.412-2.248$ \\
\hline & Constant & 2.632 & 0.813 & 10.493 & 1 & 0.001 & 0.108 & \\
\hline \multirow[t]{4}{*}{ Step 8a } & smok_stat & 1.492 & 0.456 & 10.690 & 1 & $0.001^{*}$ & 4.448 & $1.818-10.880$ \\
\hline & GI & 1.242 & 0.440 & 12.180 & 1 & $0.049^{*}$ & 2.019 & $0.933-11.579$ \\
\hline & CAL & 1.642 & 0.454 & 13.081 & 1 & $0.000^{*}$ & 5.167 & $2.122-12.584$ \\
\hline & Constant & 1.593 & 0.474 & 11.315 & 1 & 0.001 & 0.203 & \\
\hline
\end{tabular}

Cl: confidence interval; GI: gingival index; PPD: periodontal pockets depth; CAL: clinical attachment loss; BOP: bleeding on probing; Exp: expected values; ${ }^{*} P$ : statistically significant (according to multivariate regression model)

Table 3: Application of Cohran's and Mantel-Haenszel's, statistical method for controlling possible confounders

\begin{tabular}{lll}
\hline Variables & $\operatorname{Exp}(\mathbf{B})$ & \multicolumn{1}{c}{$95 \% \mathbf{C l}$} \\
\hline Gingival inflammation & & \\
$\quad$ Non-smokers & 2.137 & $0.712-4.528$ \\
$\quad$ Smokers & 4.115 & $2.027-11.842$ \\
$\quad$ Low socio-economic status & 1.896 & $0.678-3.788$ \\
$\quad$ High socio-economic status & 4.205 & $1.806-7.322$ \\
Clinical attachment loss & & \\
$\quad$ Non-smokers & 2.398 & $0.882-4.389$ \\
$\quad$ Smokers & 5.644 & $2.195-12.083$ \\
$\quad$ Low socio-economic status & 2.634 & $0.842-5.144$ \\
High socio-economic status & 4.379 & $1.765-8.047$ \\
\hline
\end{tabular}

Cl: confidence interval; Exp: expected values

examined in regard to epidemiological parameters of gender, age, educational, and socioeconomic level; however, OR's values for socioeconomic and educational level were slightly higher in the cases group compared with the controls (step 1a). Regular dental follow-up decreases the risk of PD, as this essentially reflects the level of oral hygiene; however, it has not been used in such studies. Its role would be considered indirect, i.e. non-regular dental followup contributes to dental plaque accumulation which in turn leads to PD, gingivitis, and periodontitis. No statistically significant difference between the cases and the controls was recorded regarding that parameter. Smoking is a proven risk factor for initiation and progress of both diseases, GC and $P D,{ }^{[22,24]}$ and a known confounder of cancer development. However, smoking in the cases group did not demonstrate a statistically significant difference from those in the control group. The results also revealed that the cases showed statistically significant higher values in gingival inflammation severity, according to GI, compared with the controls. These findings cannot be confirmed by previous reports as similar studies have not been performed. On the other hand, the use of the index is limited in epidemiological studies despite the fact that the index measures the inflammatory load of gingival tissue. Hujoel et al. ${ }^{[8]}$ found that gingival inflammation could be a risk factor for several types of cancer development.

No statistically significant difference was noted regarding BOP between the cases and the controls. This finding was not in accordance with the higher gingival inflammation scores recorded in the GC group. That finding existed after controlling for potential confounders such as smoking, and socioeconomic status. BOP is a critical indicator of a periodontal diagnosis, and the most reliable indicator of PD activity. ${ }^{[25]}$ Similar findings have not been reported by other investigators. A majority of the available studies have examined the possible association between PD parameters, (causal/ risk factors, and cancer development) and other PD parameters, such as tooth loss.

Also, statistically significant differences have been found between the cases and the controls, regarding clinical attachment loss (CAL) values. After assessing the ORs, the cases showed higher PPD values, compared with the controls. Similar findings have not been reported by other investigators regarding the CAL index. As already mentioned, a few studies have been carried out regarding the oral conditions in GC patients and other types of cancer. Critchlow et al., ${ }^{[26]}$ found that patients with head and neck cancer had poor oral health at the time of diagnosis. Dental caries and PD were suggested as important clinical issues.

In a similar prospective cross-sectional study, individuals with oral or oropharyngeal cancer, showed a PPD of $6 \mathrm{~mm}$ or greater in $76 \%$ of the patients evaluated, while only $10 \%$ of the patients in the control group showed the same severity of disease. The main findings of the same study was an association 
between cancer and a more severe PD regardless of oral hygiene and dental health status. ${ }^{[27]}$ Another important observation was that the GC patients showed more periodontal problems, i.e. gingival inflammation, and more PD activity in comparison with the controls. This finding should be regarded as clinically significant. Therefore it is obvious that PD is elevated in the cases group and more generalized and more severe compared to the control group. These observed differences would suggest clinical implications for the management of PD in GC patients. Based on the clinical observations that the initiation of both diseases (PD and GC) is considered a chronic inflammatory reaction, conservative periodontal therapy could reduce the biomarkers and mediators of the inflammatory response. Emphasis should be given to the preventive and strict oral hygiene of GC patients. ${ }^{[28]}$ It is important to note that the inclusion of older individuals, who had at least 20 natural teeth, could lead to an underestimation of older individuals with previous PD. These patients may have had teeth extracted for periodontal reasons.

In conclusion, PD was statistically different in patients with a GC history as expressed by parameters such as $\mathrm{GI}$ and CAL compared with healthy individuals.

\section{Authors' contributions}

Manuscript's preparation: N.A. Chrysanthakopoulos

Manuscript's review: N.A. Chrysanthakopoulos, A.A.

Oikonomou

Concept design: N.A. Chrysanthakopoulos

Literature search: N.A. Chrysanthakopoulos, A.A. Oikonomou

\section{Acknowledgments}

The authors would like to thank Dr. Jon Wagner for his contribution in language editing.

\section{Financial support and sponsorship}

None.

\section{Conflicts of interest}

There are no conflicts of interest.

\section{Patient consent}

The authors have obtained the patients' written informed consent.

\section{Ethics approval}

According to the requirements of the affiliation, this study does not require ethics approval as it was not an experimental one.

\section{REFERENCES}

1. Jemal A, Bray F, Center MM, Ferlay J, Ward E, Forman D. Global cancer statistics. CA Cancer J Clin 2011;61:69-90.

2. Kelley JR, Duggan JM. Gastric cancer epidemiology and risk factors. J Clin Epidemiol 2003;56:1-9.

3. Barr H. Gastric tumours. Medicine 2007;35:216-9.

4. Zabaleta J. Multifactorial etiology of gastric cancer. Methods Mod Biol 2012;863:411-35

5. Papapanou PN. Periodontal diseases: epidemiology. Ann Periodontol 1996;1:1-36.

6. Holmstrup P, Poulsen AH, Andersen L, Skuldbøl T, Fiehn NE. Oral infections andsystemic diseases. Dent Clin North Am 2003;47:575-98.

7. Michaud DS, Joshipura K, Giovannucci E, Fuchs CS. A prospective study of periodontal disease and pancreatic cancer in US male health professionals. J Natl Cancer Inst 2007;99:171-5.

8. Hujoel PP, Drangsholt M, Spiekerman C, Weiss NS. An exploration of the periodontitis-cancer association. Ann Epidemiol 2003;13:312-6.

9. Abnet CC, Qiao YL, Mark SD, Dong ZW, Taylor PR, Dawsey SM. Prospective study of tooth loss and incident esophageal and gastric cancers in China. Canc Caus Contr 2001;12:847-54.

10. Abnet CC, Qiao YL, Dawsey SM, Dong ZW, Taylor PR, Mark SD. Tooth loss is associated with increased risk of total death and death from upper gastrointestinal cancer, heart disease, and stroke in a Chinese population-based cohort. Int J Epidemiol 2005;34:467-74.

11. Michaud DS, Liu Y, Meyer M, Giovannucci E, Joshipura K. Periodontal disease, tooth loss, and cancer risk in male health professionals: a prospective cohort study. Lancet Oncol 2008;9:550-8.

12. Pearman T. Psychological factors in lung cancer: quality of life, economic impact, and survivorship implications. J Psychosoc Oncol 2008;26:69-80.

13. Dyszkiewicz Konwinska M, Mehr K, Owecka M, Kulczyk T. Oral health status in patients undergoing chemotherapy for lung cancer. Open J Dent Oral Med 2014;2:17-21.

14. Lavstedt S, Bolin A, Henrikson CO. Proximal alveolar bone loss in a longitudinal radiographic investigation. II. A 10-year follow-up study of an epidemiologic material. Acta Odontol Scand 1986;44:199-205.

15. Tonetti MS, Claffey N; European Workshop in Periodontology group C. Advances in the progression of periodontitis and proposal of definitions of a periodontitis case and disease progression for use in risk factor research. J Clin Periodontol 2005;32:210-3.

16. Reichert S, Stein J, Gautsch A, Schaller HG, Machulla HK. Gender differences in HLA phenotype frequencies found in German patients with generalized aggressive periodontitis and chronic periodontitis. Oral Microbiol Immunol 2002;17:360-8.

17. Loos BG, John RP, Laine ML. Identification of genetic risk factors for periodontitis and possible mechanisms of action. J Clin Periodontol 2005;32:159-79.

18. Machtei EE, Christersson LA, Grossi SG, Dunford R, Zambon JJ, Genco RJ. Clinical criteria for the definition of "established periodontitis". J Periodontol 1992;63:206-14.

19. Machuca G, Segura-Egea JJ, Jimenez-Beato G, Lakalle JR, Bullon P. Clinical indicators of periodontal disease in patients with coronary heart disease: a 10 years longitudinal study. Med Oral Patol Oral Cir Bucal 2012;17:e569-74.

20. Rubin H. Fields and field cancerization: the preneoplastic origins of cancer: asymptomatic hyperplastic fields are precursors of neoplasia, and their progression to tumors can be tracked by saturation density in culture. Bioessays 2011;33:224-31.

21. Löe H, Silness J. Periodontal disease in pregnancy. I. prevalence and severity. Acta Odontol Scand 1963;21:533-51.

22. Russell AL. Epidemiology of periodontal disease. Int Dent $J$ 1967;17:282-96. 
23. Wiebe CB, Putnins EE. The periodontal disease classification system of the American Academy of Periodontology -- an update. J Can Dent Assoc 2000;66:594-7.

24. Ferlay J, Shin HR, Bray F, Forman D, Mathers C, Parkin DM. Estimates of worldwide burden of cancer in 2008: GLOBOCAN 2008. Int J Cancer 2010;127:2893-917.

25. Lang NP, Joss A, Orsanic T, Gusberti FA, Siegrist BE. Bleeding on probing. A predictor for the progression of periodontal disease? J Clin
Periodontol 1986;13:590-6.

26. Critchlow SB, Morgan C, Leung T. The oral health status of pretreatment head and neck cancer patients. Br Dent $J$ 2014;216:E1.

27. Rezende CP, Ramos MB, Daguíla CH, Dedivitis RA, Rapoport A Oral health changes in with oral and oropharyngeal cancer. Braz J Otorhinolaryngol 2008;74:596-600.

28. Albandar JM. Epidemiology and risk factors of periodontal disease Dent Clin North Am 2005;49:517-32. 